\title{
Foreign Body Aspiration in Children: A Review of 10Years
}

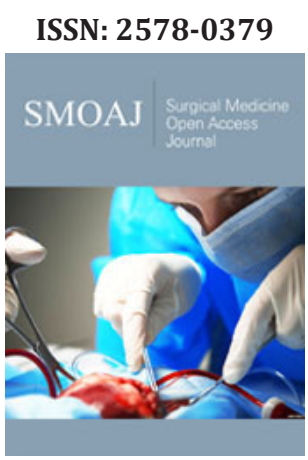

*Corresponding author: Gunther Pabst, Department of Otorhinolaryngology, Head \& Neck Surgery, Lucerne Cantonal Hospital, Switzerland

Submission: 侮June 28, 2019

Published: 㭗July 09, 2019

Volume 2 - Issue 4

How to cite this article: Pabst $\mathrm{G}^{*}$ Linder TE, Schlegel-Wagner C, Reimers $\mathrm{M}$, Pfammatter A . Foreign Body Aspiration in Children: A Review of 10Years. Surg Med Open Acc J.2(5). SMOAJ.000547.2019. DOI: 10.31031/SMOAJ.2019.02.000547.

Copyright@ Gunther Pabst, This article is distributed under the terms of the Creative Commons Attribution 4.0 International License, which permits unrestricted use and redistribution provided that the original author and source are credited.

\section{Gunther Pabst*, Christoph Schlegel-Wagner, Thomas Linder, Markus Reimers and Alain Pfammatter}

Department of Otorhinolaryngology, Head \& Neck Surgery, Lucerne Cantonal Hospital, Switzerland

\begin{abstract}
Objective: A review of all cases of suspected foreign body aspiration in children transferred to an ENT referral center for endoscopy for 10years.

Methods: In the Department of Otorhinolaryngology, Head and Neck Surgery 96 cases of endoscopy for presumed foreign body aspiration in children during the time period of 10years were retrospectively analyzed. The results were compared with a review of the actual literature.

Result: During the most recent time period in 69 out of 96 patients an aspiration was confirmed, and the foreign bodies removed. The mean patient age was 2 .3years. Organic foreign bodies, especially peanuts, were most common. In $70 \%$ of the cases endoscopy was performed within the first 24 hours. The main bronchus was most frequently affected with an almost equal distribution of both sides. Six foreign bodies were found in the esophagus.
\end{abstract}

Conclusion: When foreign body aspiration is suspected, early endoscopic removal is mandatory. In cases where no foreign body is found in the airways, esophagoscopy is necessary. In exceptional cases CT-scans can be of high diagnostic value.

Keywords: Foreign bodies; Patient; Bronchoscopy; Prevention; Esophagus

\section{Introduction}

Foreign body aspiration is a common problem in children. Toddlers 3years of age oryounger are at higher risk, since they explore their environment by putting objects preferentially in their mouths [1]. At this age the complex mechanisms of chewing and swallowing food are still underdeveloped [2]. Food, mainly nuts, beside non-organic objects are the primary causes of choking. Foreign body aspiration, especially in children requires prompt recognition and early treatment to avoid potentially serious and sometimes fatal consequences. The most reliable clinical signs of foreign body aspiration are choking and witnessed aspiration episode [3]. If foreign body aspiration is suspected, early bronchoscopy and removal is indicated as a straightforward and safe procedure [4]. In the last decade prevention of foreign body aspiration in children has become a more and more important issue [5-7].

We reviewed all cases of bronchoscopies in children for possible foreign body aspiration performed in the Department of Otorhinolaryngology, Head and Neck Surgery of the Kantonsspital Luzern in Switzerland from 2000 to 2010. The results were compared with those of a previous study from 1984 to 1994 [8]. The aim of this study was furthermore to assess the effect of a newspaper educational campaign for choking in reducing the incidence of foreign body aspiration.

\section{Material and Methods}

All bronchoscopies performed in children with suspected foreign body aspiration at a tertiary referral ENT center between 2000 and 2010 were reviewed retrospectively. Overall 96 children, mainly referred by the pediatric clinic, underwent bronchoscopy under general anesthesia using rigid Storz ventilating bronchoscopes. Type and location of the foreign body was recorded prior to removal utilizing different types of grasping forceps (Figure 1). If no foreign body could be found in the airways, esophagoscopy was carried out. 78 patients (81\%) 
underwent a routine conventional chest-X-ray prior to referral and in 3 cases a CT scan was performed preoperatively. The results were compared with a similar previous study conducted by the same Department during the years 1984 to 1994 [8]. Binary classification tests have been used to calculate sensitivity, specificity, positive and negative predictive values and accuracy. For prevention articles have been published in teamwork with the main local newspaper every year from 1994 on, to educate and warn especially parents of young children but as well the public in general and family doctors of choking hazards. The focus was annually on the pre-Christmas period and peanuts. In Switzerland peanuts are, as in many other countries, the most common aspirated foreign bodies and mainly consumed in the pre-Christmas period. In addition, information leaflets about choking hazards were hanged up in the outpatient clinic of our Department.

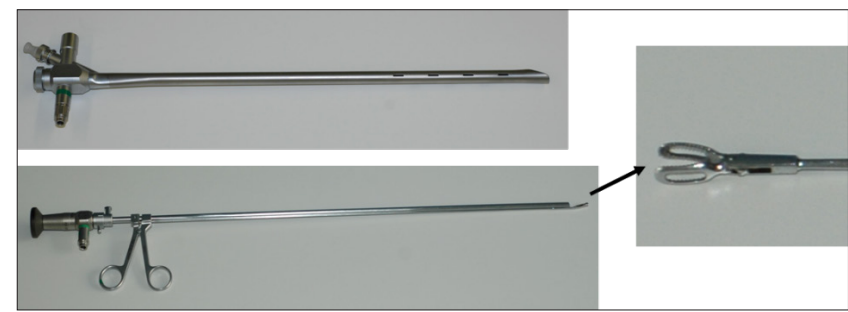

Figure 1: Storz rigid bronchoscope (upper left picture). Endoscopic optic with grasping forceps made especially for removal of peanuts.

\section{Result}

Overall 96 children underwent bronchoscopy for suspicion of foreign body (FB) aspiration during the time period 2000 to 2010. The mean patient age was $2.3 y e a r s$, with a range from a few months up to 15years (Figure 2). In 69 of total 96 patients (72\%) a foreign body could be found and removed during endoscopy. In most cases the foreign body was located in the main bronchus, with a nearly equal distribution (28 in the right and 25 in the left). The remaining FB were located in the trachea $(n=2)$, middle lobe $(n=3)$, left lower lobe $(n=2)$, pharynx $(n=1)$, larynx $(n=2)$ and esophagus $(n=6)$. This distribution is illustrated in Figure 3. In 83\% $(n=57)$ the foreign bodies were of organic and in $17 \%(n=12)$ non-organic nature. The most common FBs were peanuts in 35 cases (51\%). During endoscopic removal, the peanut often broke into several smaller pieces which is why no comment can be made about the initial number of peanuts in the airways. In the majority of patients ( $n=48,70 \%$ ) bronchoscopy was performed within the first 24 hours after the incident. In the remaining $30 \%$ of patients FB removal was delayed (later than 24hours) mainly because of the parents (in 75\%). Most of the patients stayed postoperatively overnight for observation purposes and were dismissed the following day. In $81 \%$ of the cases $(n=78)$ a conventional chest X-ray has been performed preoperatively. The Sensitivity was $77 \%$, Specificity $78 \%$, positive predictive value $91 \%$, negative predictive value $46 \%$ and accuracy $73 \%$. In three exceptional cases a CT scan has been performed (Figure 4).

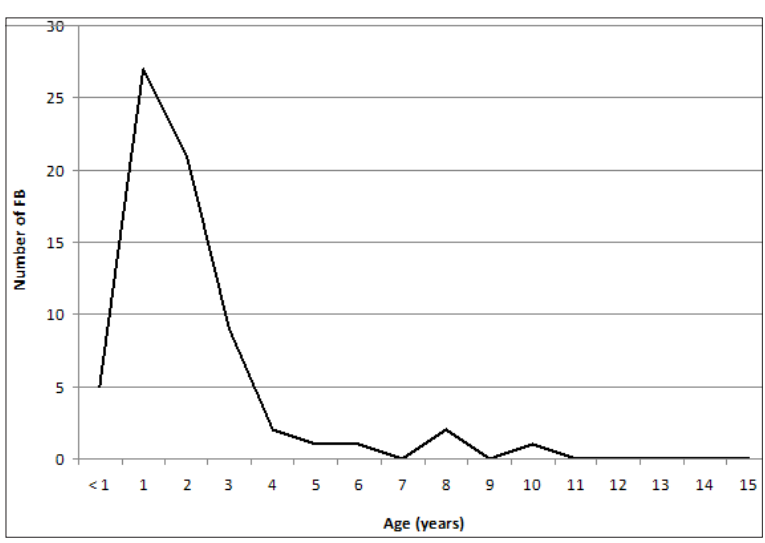

Figure 2: Age distribution of patients with foreign body $(\mathrm{FB})$ aspiration.

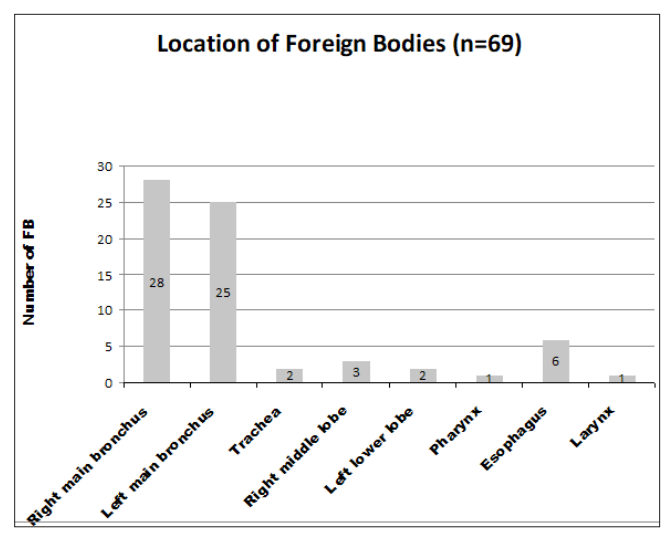

Figure 3: Showing the anatomical distribution of foreign bodies. Surprisingly 6 foreign bodies were removed from the esophagus.
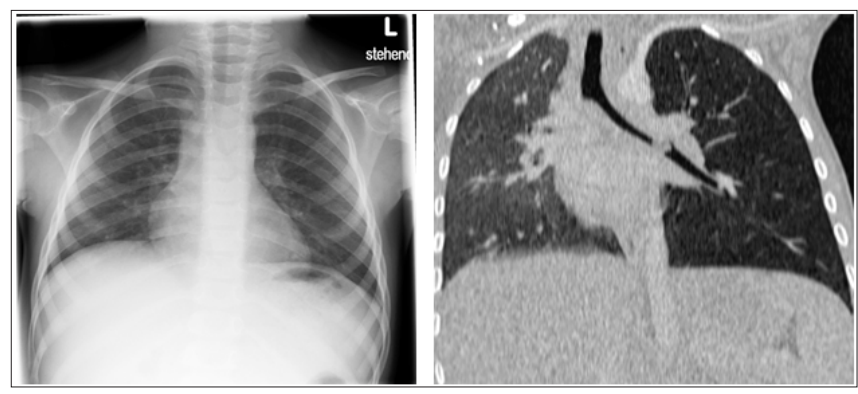

Figure 4: The right picture shows a conventional $\mathrm{X}$-ray of a 3-year-old boy with suspected foreign body aspiration. No direct and indirect signs for an aspirated foreign body are present. The left picture shows a chest CT scan of the same patient with the foreign body (peanut) clearly visible in the left main bronchus.

The results of the present study and of a similar previous study performed in our Department over the time period 1984 to 1994 are shown and summarized in Table 1. Compared to the previous study over the same 10-year time period, 68 times less often endoscopies for foreign body removal had to be carried out. The peanuts, as the most common foreign bodies, were $20 \%$ less frequent. 
Table 1: Showing characteristics of patients undergoing bronchoscopy for suspicion of foreign body aspiration during the period of 2000-2010 (present study) and 1984-1994 (previous study).

\begin{tabular}{|c|c|c|c|}
\hline & Previous study 1984-1994(n=164) & Present study 2000-2010 (n=96) & Reduction \\
\hline Median age (years) & 3.1 & 2.3 & \\
\hline Foreign body & 164 & 96 & \\
\hline Present & $90(55 \%)$ & $69(72 \%)$ & $21(23 \%)$ \\
\hline Absent & $74(45 \%)$ & $27(28 \%)$ & $47(64 \%)$ \\
\hline organic & $78(87 \%)$ & $57(83 \%)$ & $21(27 \%)$ \\
\hline an organic & $12(13 \%)$ & $12(17 \%)$ & 0 \\
\hline Peanuts & $44(49 \%)$ & $35(15 \%)$ & $9(20 \%)$ \\
\hline \multicolumn{4}{|l|}{ Location of Foreign Body } \\
\hline Right main bronchus & $34(38 \%)$ & $28(41 \%)$ & $6(18 \%)$ \\
\hline Left main bronchus & $42(47 \%)$ & $25(36 \%)$ & $17(40 \%)$ \\
\hline Other Locations & $14(15 \%)$ & $16(23 \%)$ (6 in Oesophagus) & \\
\hline \multicolumn{4}{|l|}{ Time of Bronchoscopy } \\
\hline within first $24 \mathrm{~h}$ & $48(53 \%)$ & $48(70 \%)$ & $17 \%$ \\
\hline after first $24 \mathrm{~h}$ & $42(47 \%)$ & $21(30 \%)$ & $21(50 \%)$ \\
\hline
\end{tabular}

\section{Discussion}

Reviewing 20years of endoscopic removal of aspirated foreign bodies in children, the most common foreign body at our clinic is the peanut with approximately $50 \%$. Mainly children 1-3years of age were affected (Figure 2). The foreign bodies were located in the main bronchus with an almost equal distribution (Figure 3). This can be explained by the fact, that children unlike adults have an equal diameter and Broncho-tracheal angle of the main bronchi. From 2000 to 2010 six foreign bodies were found in the esophagus despite clinically suspected aspiration. Therefore, we advise to perform an esophagoscopy in addition to the bronchoscopy, especially when no foreign body can be found in the tracheobronchial system.

Compared to the previous study (1984-1994) only 96 children with high suspicion of foreign body aspiration instead of 164 have been transferred to our Department for endoscopy from 2000 to 2010. During endoscopy foreign bodies were found and removed in $72 \%$ of the cases. The peanut was still the most common foreign body with $50 \%$ frequency, but, compared to the previous study, $20 \%$ less frequent. So, a decrease in the total amount of foreign body and peanut aspirations during the second time period has been noted. We are convinced, that this decrease is mainly due to our prevention campaign described above.

Other studies have been investigating the effect of educational campaigns in preventing foreign body aspirations in children before Sadan N. et al. [6] presented in Israel a successful reduction of FB aspiration by $35 \%$ with a nationwide educational campaign through the media and in community pediatric care centers [7]. On the island of Crete (Greece) Karatzanis AD et al. [5] showed as well a decrease of FB aspirations in children thanks to a broad media campaign, information leaflets for the public and educational lectures in schools. In India Singh A et al. [8] demonstrated that
$25 \%$ of the Indian public community is not aware of foreign body aspiration in childhood [9]. Fortunately, around the world more and more progress is made in preventing foreign body aspiration in children. The American Academy of Pediatrics set up a policy statement about prevention of choking among children [10].

From 2000 to 2010 endoscopy and FB removal was performed within the first 24 hours in $70 \%$ of the cases compared to $53 \%$ in the previous study episode (1984-1994). Delayed endoscopy after the first 24 hours is mainly due to the parental negligence. The higher percentage of early presentation of children with foreign body aspiration might be thanks to the prevention newspaper campaign informing and warning the parents about choking hazards. It is well known that delay of foreign body removal leads to higher morbidity and secondary injuries like for example pneumonia, atelectasis and obstructive emphysema. Mu L et al. [11] also showed that the complication rate rises with longer aspiration time. Therefore, aspirated foreign bodies have to be removed endoscopically as quickly as possible. The most important sign of foreign body aspiration is a typical history of witnessed aspiration episode with choking and coughing [3]. Beside clinical examination with auscultation and percussion often a conventional radiography is performed. Reviewing our present study, in $81 \%$ of the cases with suspected foreign body aspiration (FBA) in children a chest X-ray has been performed before endoscopy.

In our study Chest X-rays rarely displayed the foreign body itself as most FBs are not radiopaque. Usually indirect signs like hyperlucent lung and post obstructive atelectasis or infiltrates indicate FBA. Therefore, CT scan was performed in 3 unusual cases preoperative and revealed the foreign body with its exact location and dimension. In all these 3 subjects patient's history and/or clinical presentation was not typical for foreign body aspiration and previous conventional radiographs showed no pathology (Figure 4) 
Bai W et al. [10] compared in 45 children with FBA the CT and chest X-ray findings. All of the foreign bodies $(100 \%)$ were identified by chest CT. In contrary $52.4 \%$ of the patients with tracheobronchial FB showed no abnormalities on conventional X-ray. Six foreign bodies were found in the esophagus despite clinically suspected aspiration. Therefore, we advise to perform an esophagoscopy in addition to the bronchoscopy, especially when no foreign body can be found in the tracheobronchial system.

\section{Conclusion}

Foreign body aspirations in children continue to be a challenging diagnosis. The foreign bodies were mainly located in the main bronchi with a nearly equal distribution. In our patient collective the peanut was the most common foreign body removed. In some cases of suspected FBA foreign bodies were found in the esophagus. Therefore, we advise to perform an esophagoscopy, especially if no foreign body is found in the tracheobronchial system. Because chest X-rays rarely displayed the foreign body itself, CT scans can be very useful in exceptional cases where patient's history, clinical presentation and course are not typical but ominous for foreign body aspiration to detect the FB and help the surgeon plan for endoscopic removal.

Prevention by the newspaper and information leaflets, as shown in this report, can help to decrease the incidence of foreign body aspiration.

\section{References}

1. Smith SA, Norris B (2003) Reducing the risk of choking hazards: Mouthing behavior of children aged 1 month to 5years. Inj Control Saf Promot 10(3): 145-154.
2. Carruth BR, Skinner JD (2002) Feeding behaviors and other motor development in healthy children (2-24 months). J Am Coll Nutr 21(2): 88-96.

3. Orji FT, Akpeh JO (2010) Tracheobronchial foreign body aspiration in children: How reliable are clinical and radiological signs in the diagnosis? Clin Otolaryngol 35(6): 479-485.

4. Black RE, Johnson DG, Matlak ME (1994) Bronchoscopic removal of aspirated foreign bodies in children. J Pediatr Surg 29(5): 682-684.

5. Karatzanis AD, Vardouniotis A, Moschandreas J, Prokopakis EP, Michailidou E, et al. (2007) The risk of foreign body aspiration in children can be reduced with proper education of the general population. Int J Pediatr Otorhinolaryngol 71(2): 311-315.

6. Sadan N, Raz A, Wolach B (1995) Impact of community educational programmes on foreign body aspiration in Israel. Eur J Pediatr 154(10): 859-862.

7. Reimers M, Schlegel Ch. Tracheobronchial foreign bodies in children: A retrospective analysis. ORL, Current Problems of Otorhinolaryngology (19): 292-298.

8. Singh A, Ghosh D, Samuel C, Bhatti W (2010) Pediatric foreign body aspiration: How much does our community know? J Indian Assoc Pediatr Surg 15(4): 129-132.

9. (2010) Committee on injury, violence, and poison prevention of the American academy of pediatrics. Policy Statement-Prevention of choking among children. Pediatrics 125(3): 601-607.

10. Bai W, Zhou X, Gao X, Shao C, Califano JA, et al. (2011) The value of chest CT in the diagnosis and management of tracheobronchial foreign bodies. Pediatr Int 53(4): 515-518.

11. Mu L, He P, Sun D (1991) The causes and complications of late diagnosis of foreign body aspiration in children. Report of 210 cases. Arch Otolaryngol Head Neck Surg 117(8): 876-879. 